\title{
Clinical Trials
}

\section{Effect of Delayed Cord Clamping on Neurodevelopment at 3 Years: A Randomized Controlled Trial}

\author{
Johan Henrik Martin Berg ${ }^{a, b}$ Manuela Isacson ${ }^{a, c}$ Omkar Basnet ${ }^{d}$ \\ Rejina Gurung ${ }^{d}$ Kalpana Subedi ${ }^{\mathrm{e}}$ Ashish KC $^{f}$ Ola Andersson ${ }^{\mathrm{a}, \mathrm{g}}$
}

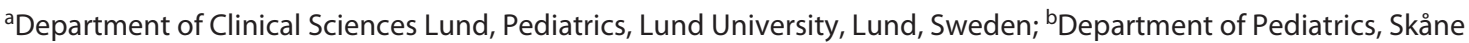
University Hospital, Malmö/Lund, Sweden; 'Sachs' Children and Youth Hospital, South General Hospital, Stockholm, Sweden; dGolden Community, Lalitpur, Nepal; eParopakar Maternity and Women's Hospital, Kathmandu, Nepal; fDepartment of Women's and Children's Health, Uppsala University, Uppsala, Sweden; 9Department of Neonatology, Skåne University Hospital, Malmö/Lund, Sweden

\section{Keywords}

Ages and Stages Questionnaire · Delayed cord clamping · Neurodevelopment · Umbilical cord · Child

\begin{abstract}
Introduction: Iron deficiency (ID) is associated with poor neurodevelopment. We have previously shown that delayed umbilical cord clamping (CC) improves iron stores at 8 months and neurodevelopment at 1 year in term, healthy infants in Nepal. Objective: The aim of this study was to assess the effects of delayed CC ( $\geq 180 \mathrm{~s}$ ) compared to early CC ( $\leq 60 s$ ) on neurodevelopment using the Ages and Stages Questionnaire (ASQ) at age 3 years. Methods: In 2014, 540 healthy Nepalese infants born at term were randomized in a 1:1 ratio to delayed or early CC. At 3 years of age, ASQ assessment was performed by phone interviews with parents. A score $>1$ standard deviation below the mean was defined as "at risk" for developmental impairment. Results: At 3 years of age, 350 children were followed up, $170(63.0 \%)$ in the early CC group and $180(66.7 \%)$ in the delayed CC group. No significant differences in ASQ scores in any domains between groups were found. However, more girls were "at risk" for affected gross motor development in the early CC group:
\end{abstract}

14 (18.9\%) versus 6 (6.3\%), $p=0.02$. Conclusion: There were no significant differences in ASQ scores in any domains between groups. In the subgroup analysis, fewer girls who underwent delayed CC were "at risk" for delayed gross motor development. Due to the pronounced difference in iron stores at 8 months postpartum in this cohort, follow-up studies at an older age are motivated since neurodevelopmental impairment after early ID may be more detectable with increasing age.

(C) 2021 The Author(s)

Published by S. Karger AG, Basel

\section{Introduction}

Iron deficiency (ID) is the most common nutrient deficiency globally and is most prevalent in children of age $0-5$ years [1]. Iron has an important role in brain development and especially during the first 3 years of life, ID may lead to irreversible neurodevelopmental abnormalities [2].

By waiting to clamp the umbilical cord at birth, the infant's blood volume can be increased by $30-40 \%$ (25-35 $\mathrm{mL} / \mathrm{kg}$ ), which provides a substantial iron addition [3]. Delayed umbilical cord clamping (CC), usually defined as

Correspondence to:

Johan Henrik Martin Berg, johan.berg.449@ hotmail.com

karger@karger.com www.karger.com/neo

Karger $\stackrel{\text { ' }}{5}$

GOPEN ACCESS
(C) 2021 The Author(s)

Published by S. Karger AG, Basel

This is an Open Access article licensed under the Creative Commons Attribution-NonCommercial-4.0 International License (CC BY-NC) (http://www.karger.com/Services/OpenAccessLicense), applicable to the online version of the article only. Usage and distribution for commercial purposes requires written permission. 
$>60-180 \mathrm{~s}$ after birth, compared to early CC, usually defined as $<30 \mathrm{~s}$ after birth, has been shown to decrease the incidence of ID up to 8 months after birth and improve hemoglobin from birth up to 1 year of age in term infants [4-6].

In a Swedish randomized controlled trial, 4-year-old children had higher scores in the fine motor and social domains of the Ages and Stages Questionnaire (ASQ) when CC was performed $\geq 3 \mathrm{~min}$ after delivery compared to $\leq 10 \mathrm{~s}$ after delivery [7]. Other studies have indicated improved brain myelin content at 4 months and 1 year following delayed CC compared to early CC $[8,9]$.

In our original studies in this cohort, we have reported improved ferritin levels at 8 months and higher neurodevelopment scores at 1 year when $\mathrm{CC} \geq 3$ min was compared to $C C \leq 1$ min postpartum in Katmandu, Nepal [6, 10]. Nepal is a low-income country with high neonatal mortality rates, especially among the poorest [11]. At the time of the original intervention, institutional birth coverage in Katmandu was high (93.8\%), and 6.7\% children under-five were underweight [12]. High levels of ID have previously been reported in our study population [6].

The aim of the present study was to assess neurodevelopment using the ASQ in the same cohort at 3 years of age. We hypothesized that neurodevelopmental outcome measured by the ASQ would remain improved in the delayed CC group compared to the early CC group.

\section{Methods}

\section{Study Setting, Design, and Participants}

This study presents the follow-up at 3 years of age as a secondary outcome of a randomized controlled trial conducted in the Paropakar Maternity and Women's Hospital, a government-funded tertiary hospital located in Katmandu, Nepal. The original study was conducted at the Maternal and Neonatal Service Centre which was the hospital's low-risk delivery unit.

Two parallel groups were compared in a 1:1 ratio, early CC ( $\leq 60 \mathrm{~s})$ and delayed CC ( $\geq 180 \mathrm{~s})[6,10]$. The Institutional Review Board of the hospital and the Ethical Review Board of Nepal Health Research Council approved the study (2014:1403).

The inclusion criteria were uncomplicated pregnancies, no complication at admission and healthy mothers (no clinical history of hypertension, infection, diabetes, or any chronic medical condition), expected vaginal delivery, gestational age of 34-41 weeks, and singleton pregnancy. The criteria were identical to the admission criteria of the delivery unit. Enrollment was performed by research assistants present day and night by obtaining written consent from the women eligible and willing to participate.

\section{Randomization}

A random sequence was generated using Microsoft Excel (Microsoft, Seattle, WA, USA). The treatment allocation was revealed when delivery was imminent as described in our previous study [6]. The research team involved in collecting or administrating the ASQ interviews was blinded to the allocation measures.

\section{Outcome}

As reported previously, the primary outcome of the original study was anemia levels at 8 months of age [6]. The secondary outcomes were ferritin level at 8 months and 1 year, hemoglobin level at 1 year, and neurodevelopment using ASQ at age 1 year $[6,10]$. In this study, we report follow-up of neurodevelopment at age 3 years (ClinicalTrials.gov Identifier: NCT02222805: https://clinical-trials.gov/ct2/show/NCT02222805). The outcomes assessed were ASQ total score, and scores on the 5 domains are listed below.

\section{Measure}

The third edition of the ASQ is a series of parent-completed screening tools which measures developmental milestones. The ASQ consists of 3 sections: (1) Brief demographic items, (2) thirty questions divided into 5 different domains (communication, fine motor, gross motor, problem-solving ability, and personal-social functioning), and (3) 10 open-ended questions regarding parental concerns about their children's abilities and possible medical problems. In the second and main section, there are 3 choices for each question: "Yes," "Sometimes," and "Not yet," which score 10, 5 , and 0 , respectively. Domain scores are then obtained by the sum of each item. The protocol's specified inclusion age range, 34 months +16 days -38 months +30 days, was used. A total score was obtained by summing up the domain scores. This score has shown the best sensitivity and specificity when screening for developmental outcome [13]. An "at risk" cutoff score of $<1$ standard deviations below the mean was added to the $<2$ standard deviation threshold specified by the manual to increase sensitivity [14]. This has also been done in other studies $[13,15]$.

\section{Procedures}

For the 3-year follow-up, the English questionnaire of the 36-month ASQ 3rd edition was first translated to Nepali and then the Nepali version was cross-checked with the English version by a professional English translator. The questionnaire was then pilot tested on 10 children at an age similar to the study population. Due to high levels of illiteracy in Nepal, it was not possible to obtain written responses even though the ASQ is a parent report questionnaire. Instead, 2 nurses trained on using the ASQ guidelines conducted the interviews by phone. As described in our previous publication, parents were interviewed regarding the feeding habits of the infant (e.g., what type of food had been given and frequency of meals) at age 8 and 12 months [6].

\section{Statistical Analysis}

For the primary outcome of the original trial, sample size was estimated on a difference of hemoglobin levels at 8 months. For variables with normal distribution, unpaired student's $t$ test was used. Fischer's exact test was used for categorical variables. Spearman's rank correlation coefficient was used for investigating correlation between ASQ "at risk" scores at 1 and 3 years. The following criteria were used for choosing variables to include in regression analysis: (a) imbalanced baseline data (significant difference between groups, $p<0.05$ ) and (b) a significant correlation between the outcome variable and the baseline variable. SPSS (version 25; IBM Corp., New York, NY, USA) was used for statistical calculations. 


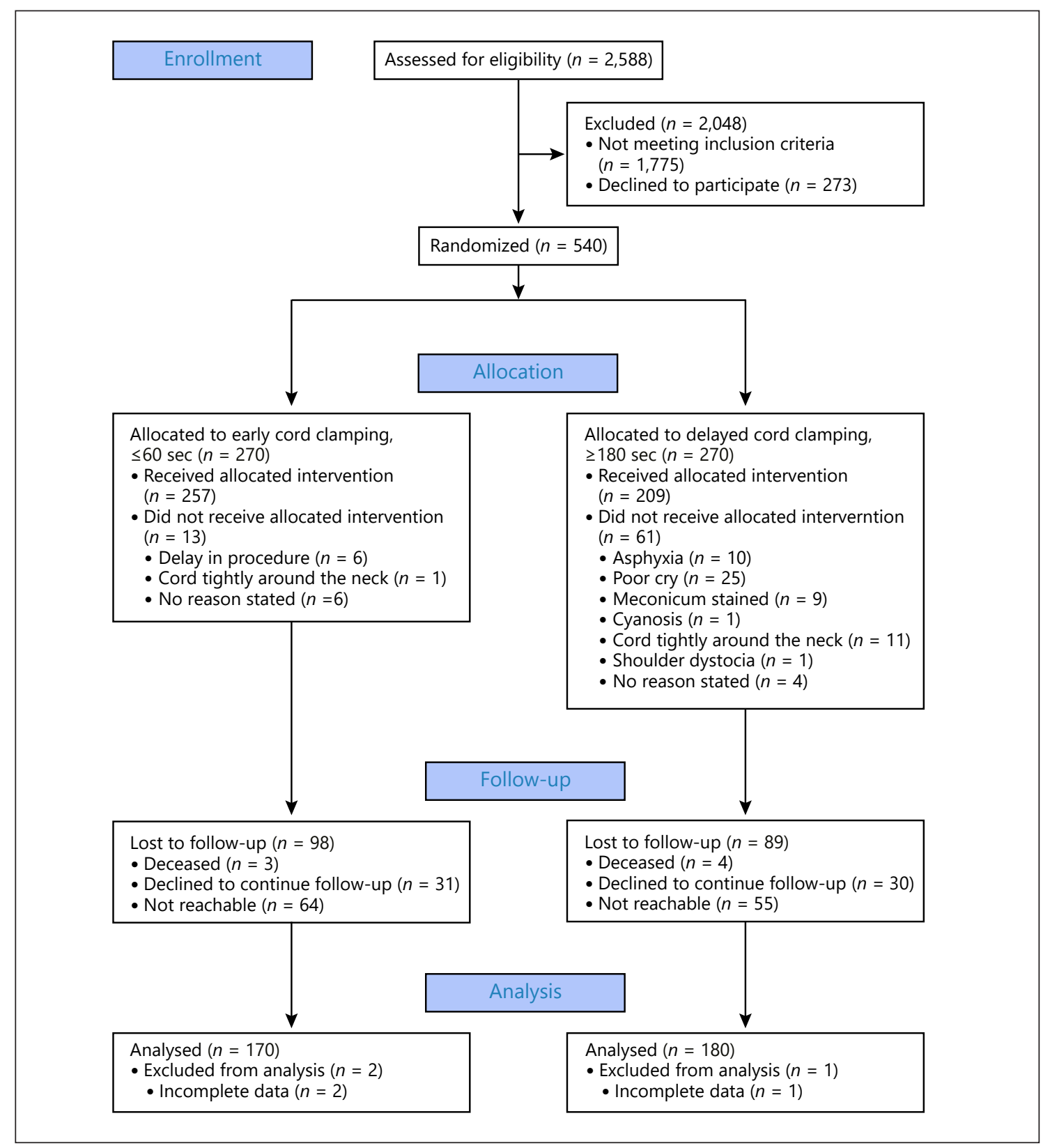

Fig. 1. Flow diagram for randomized clinical trial of delayed versus early umbilical CC. CC, cord clamping.

\section{Results}

Between October 2 and November 21, 2014, 540 newborns were included in the study. They were randomized to early CC $(n=270)$ or delayed CC $(n=270)$. At 3 years, $170(63.0 \%)$ in the early CC group and $180(66.7 \%)$ in the delayed CC group were analyzed. In these children, CC had been performed according to protocol in 163 (95.9\%) versus $138(76.7 \%)$ in the early versus delayed CC group.
Reasons for protocol deviations and lost to follow-up are listed in Figure 1.

There were no significant differences in maternal and neonatal baseline data between groups except for a higher gestational age in the delayed CC group ( $39.0 \pm 1.2$ vs. $39.3 \pm 1.1$ weeks, $p=0.006$ ) (Table 1 ). The difference was no longer significant when excluding the infants who did not receive the allocated intervention. When comparing food consumption between groups at age 8 and 12 
Table 1. Baseline characteristics of mothers and infants according to the intervention

\begin{tabular}{|c|c|c|c|c|}
\hline & \multicolumn{4}{|c|}{ Umbilical CC group } \\
\hline & \multicolumn{2}{|c|}{ early CC $(\leq 60 \mathrm{~s}, n=170)^{\mathrm{a}}$} & \multicolumn{2}{|c|}{ delayed CC $(\geq 180 \mathrm{~s}, n=180)^{\mathrm{a}}$} \\
\hline & mean $\pm S D$ & $\begin{array}{l}\text { participants, } \\
n\end{array}$ & mean \pm SD & $\begin{array}{l}\text { participants, } \\
n\end{array}$ \\
\hline \multicolumn{5}{|l|}{ Mother } \\
\hline Age, years & $24.6 \pm 4.5$ & 170 & $23.9 \pm 3.8$ & 180 \\
\hline Parity (including study child) & $1.7 \pm 0.9$ & 170 & $1.7 \pm 0.9$ & 180 \\
\hline Antenatal checkups ${ }^{c}$ & $4(0-8)$ & 156 & $4(0-8)$ & 169 \\
\hline \multicolumn{5}{|l|}{ Infant } \\
\hline Gestational age, weeks ${ }^{\mathrm{b}}$ & $39.0 \pm 1.2$ & 170 & $39.3 \pm 1.1$ & 180 \\
\hline Birth weight, g & $3,056 \pm 445$ & 170 & $3,027 \pm 414$ & 180 \\
\hline Apgar score, $1 \mathrm{~min}^{\mathrm{c}}$ & $6(1-7)$ & 170 & $6(1-7)$ & 178 \\
\hline Apgar score, $5 \min ^{\mathrm{c}}$ & $8(2-9)$ & 170 & $8(2-9)$ & 178 \\
\hline Time to CC, s & $35.4 \pm 20.0$ & 170 & $157.9 \pm 72.1$ & 180 \\
\hline
\end{tabular}

Table 2. ASQ results at 3 years in infants randomized to delayed versus early umbilical cord clamping

\begin{tabular}{lllll}
\hline & \multicolumn{1}{l}{ CC } & & $\begin{array}{l}\text { Mean difference } \\
\text { (95\% CI) }\end{array}$ & $p$ value $^{\mathrm{a}}$ \\
\cline { 2 - 4 } & $\begin{array}{l}\text { early CC }(\leq 60 \mathrm{~s}, n=170) \\
\text { ASQ score }(\text { mean } \pm \mathrm{SD})\end{array}$ & $\begin{array}{l}\text { delayed CC }(\geq 180 \mathrm{~s}, n=180) \\
\text { ASQ score }(\text { mean } \pm \mathrm{SD})\end{array}$ & \\
\hline Total & $269.8 \pm 25.9$ & $270.8 \pm 26.2$ & $-1.0(-6.5$ to 4.5$)$ & 0.72 \\
Communication & $57.2 \pm 5.3$ & $57.4 \pm 4.7$ & $-0.2(-1.3$ to 0.8$)$ & 0.69 \\
Gross motor & $54.5 \pm 8.8$ & $55.4 \pm 7.8$ & $-0.9(-2.7$ to 0.8$)$ & 0.30 \\
Fine motor & $54.8 \pm 8.8$ & $54.7 \pm 9.9$ & $0.1(-2.0$ to 2.2$)$ & 0.93 \\
Problem solving & $55.5 \pm 6.8$ & $55.3 \pm 7.4$ & $0.2(-1.3$ to 1.7$)$ & 0.80 \\
Personal-social & $47.8 \pm 7.7$ & $48.0 \pm 8.0$ & $-0.2(-0.8$ to 1.8$)$ & 0.83 \\
\hline
\end{tabular}

ASQ, Ages and Stages Questionnaire; CC, cord clamping; SD, standard deviation. ' $p$ value by Student's $t$ test.

months, more children in the early CC group received cow's milk at age 8 months than in the delayed CC group: $14(10.5 \%)$ versus $5(3.2 \%)(p=0.03)$. No group difference was found in the consumption of eggs, cereal porridge, soup, meat, breast milk at age 8 and 12 months, or cow's milk at age 12 months between groups (data not shown). ASQ assessment was performed at the mean age of 1,118.2 \pm 19.5 days in the early CC group and $1,116.1 \pm 17.1$ days in the delayed CC group $(p=0.56)$. Sex distribution was similar in both groups.

No significant differences in ASQ scores were found between groups (Table 2), and there were no significant differences when analyzing the ASQ scores for boys and girls separately. The proportion of children having an ASQ "at risk" score did not differ significantly between the groups (see online suppl. Table 1; for all online suppl. material, see www.karger.com/doi/10.1159/000515838). There were significantly more girls "at risk" for gross motor developmental delay in the early than in the delayed CC group: $14(18.9 \%)$ versus $6(6.3 \%)(p=0.02)$.

\section{Adjusted Analysis}

No baseline variable was correlated to ASQ total score. As there was a significant difference in gestational age and cow's milk consumption at 8 months between groups, we also analyzed correlation to the ASQ domains. A small correlation between ASQ personal-social score and gestational age was found (Spearman's $\rho=0.11 p=0.04$ ). After adjusted analysis including gestational age, the results in personal-social score did not change. 


\section{Auxiliary Analysis}

ASQ "at risk" scores at 1 and 3 years did not correlate significantly (results not shown). No difference was found in ASQ scores at 3 years in children with and without ID anemia at 8 months. Children without ID anemia at 8 months scored higher at 3 years in the personal-social domain, but this difference was not significant (48.1 \pm 7.4 vs. $46.1 \pm 8.6, p=0.06$ ).

Due to the high attrition rate, we compared baseline neonatal and maternal characteristics for the infants analyzed at 3 years with the infants lost to follow-up. The mothers in the lost to follow-up group were significantly younger ( $22.4 \pm 3.8$ vs. $24.2 \pm 4.1$ years, $p=0.001)$. Maternal age was not correlated to ASQ scores at 3 years of age. Otherwise, there were no significant differences between groups in number of pregnancies, gestational age, sex, Apgar score at 1 and $5 \mathrm{~min}$, birth weight, or time to umbilical CC.

\section{Discussion}

To our knowledge, this is the first randomized controlled trial to assess neurodevelopment in term infants comparing early and delayed CC at age 3 years. Higher ferritin levels at 8 months and better developmental scores assessed by ASQ at 1 year following delayed CC were previously reported in this study population. Our hypothesis was that neurodevelopmental outcome scores would remain higher in the delayed CC group at 3 years.

We found no difference in ASQ scores at age 3 years between the children delivered with early or delayed CC. There was no correlation between the ASQ "at risk" scores in our study and the scores in the study at age 1 year. No difference in 3-year ASQ scores comparing the children with and without ID anemia at 8 months postpartum was seen. When analyzing the results according to children's sex, no significant differences between the randomized groups were found, except that significantly fewer girls in the delayed CC group were "at risk" for impaired gross motor development.

The lack of strong differences between groups that could be attributable to that ID might have caused neurodevelopmental changes that need a test more targeted to specific cognitive domains [16]. Also, neurodevelopmental assessment scales may correlate poorly over time, especially when done before 12-18 months of age. This could explain why the ASQ scores did not correlate between age 1 and 3 years $[17,18]$.
A trend toward positive neurodevelopmental effects following delayed CC, more pronounced in boys, was seen in Swedish, term babies at 4 months, 1 , and 4 years $[7,19,20]$. Timing of CC affected different developmental domains at different ages in our cohort as well as in the Swedish cohort. An explanation could be that the functional presentation of ID can differ with time as the brain develops and new skills are acquired $[2,16]$.

Improved motor outcome after delayed CC was seen in preterm boys at age 7 months and in both sexes at 1822 months as well as in term, 2-year-old children undergoing intact cord resuscitation [21-23]. In our study, there was an effect on gross motor development only in girls. Preterm girls had better motor and language outcome than that of boys following either umbilical cord milking or delayed CC at age 3.5 years [24].

ID early in life affects formation and myelination of axons, neurotransmitter signaling, gene expression, and dendritogenesis in the developing brain. The location and extent of these effects depend on the duration, severity, and at which age the ID occurs [2]. Recently, Mercer et al. $[8,9]$ showed that delayed CC was associated with a higher degree of brain myelin on magnetic resonance imaging at age 4 months and 1 year, including areas involved in motor function. This was an area where we found a group difference in neurodevelopment scores among girls.

Finally, the intervention might have had no effect on neurodevelopment. However, several studies have shown that early ID has irreversible negative neurodevelopmental consequences. In fact, the effects of early ID increase with age and may become more easily assessable as the child grows older $[2,16]$. As we did show pronounced differences in iron stores at 8 months in this cohort previously, later impact on neurodevelopment was expected. It is therefore motivated to do further follow-up studies on psychomotor development, cognitive performance, or school performance in these children.

\section{Limitations}

The high attrition rate in our study (early CC group: $37.0 \%$ and delayed CC group: $33.3 \%$ ) and the high level of protocol deviation $(23.3 \%)$ in the delayed CC group may have influenced our results. Yet, when we excluded the protocol deviations from the analysis, the results remained unchanged (online suppl. Table 2).

Our study was powered to find a difference in hemoglobin levels at 8 months postpartum, not to find a difference in neurodevelopment and the subgroup analysis regarding ASQ scores divided by sex was based on a small number of "at risk" children. This could have affected our 
results. In a post hoc analysis, 260 children would have been needed in each randomized group to show a significant group difference in gross motor "at risk" scores in both sexes.

Other researchers have used the same questionnaire in Nepal but as a direct observer tool by a professional [25]. In our study, the ASQ assessments were done by phone interviews. This has not been done before in Nepal and may have caused a social desirability bias. Moreover, only healthy infants were included which could limit the applicability of the ASQ since it is designed to screen for developmental delay. Both circumstances may explain the higher ASQ mean values in our study compared to the study mentioned above [25]. Even so, the biases in our study should be equally distributed between the groups due to the randomized design.

According to a recent review, the Caregiver-Reported Early Child Development Instruments may be a more suitable population screening tool in low- and middleincome countries [26]. Since the ASQ has been shown to be compatible across a wide range of cultures, languages, and countries, we still think it is a good option for further studies in similar settings [27].

\section{Conclusion}

We found no significant overall difference in neurodevelopment assessed by the ASQ in 3-year-old children who were randomized to delayed CC or early CC. Fewer girls in the delayed CC group were "at risk" of gross motor developmental delay. Studies examining the neurodevelopmental effects of early ID suggest that abnormalities increase with time. Thus, to further understand the effect of delayed CC on neurodevelopment, follow-up studies on cognition and development are required at later ages.

\section{Acknowledgements}

We thank all contributing staff members at Paropakar Maternity and Women's Hospital and at Golden Community Nepal. We also thank the former director of Paropakar and Maternity and Women's Hospital, Prof. Jageshwar Gautam, Dr. Sheela Verma, former matron Maiya Manandhar, and Nisha Rana.

\section{Statement of Ethics}

Ethical approval was obtained from the Institutional Review Board of the hospital and the Ethical Review Board of Nepal Health Research Council (reg. no. 2014:1403). The original study was registered at clinicaltrials.gov NCT02222805, 2014 Aug 21.

Informed written consent of the caregivers willing to participate was obtained in the original study. The caregivers were called by phone and verbal consent was obtained from them for the assessments in the present study, which took place between October 2017 and January 2018.

\section{Conflict of Interest Statement}

The authors have no conflicts of interest relevant to this article to disclose.

\section{Funding Sources}

This study was supported by grants from the Golden Community, Nepal, the Swedish Society of Medicine, the Little Child's Foundation (Sweden), the Swedish Society of Medical Research, and the United Nations Children's Fund.

\section{Author Contributions}

Dr. Berg carried out the initial biostatistical analyses, drafted the initial manuscript, and reviewed and revised the manuscript. Ms. Gurung, Mr. Basnet, and Dr. Subedi coordinated and supervised the data collection and reviewed and revised the manuscript. Drs. KC and Isacson conceptualized and designed the study and reviewed and revised the manuscript. Dr. Andersson obtained funding, conceptualized and designed the study, carried out the initial biostatistical analyses, and reviewed and revised the manuscript. All authors approved the final manuscript as submitted and agree to be accountable for all aspects of the work.

\section{References}

1 McLean E, Cogswell M, Egli I, Wojdyla D, de Benoist B. Worldwide prevalence of anaemia, WHO vitamin and mineral nutrition information system, 1993-2005. Public Health Nutr. 2009 Apr;12(4):444-54.

2 Georgieff MK. Long-term brain and behavioral consequences of early iron deficiency. Nutr Rev. 2011 Nov;69(Suppl 1):S43-8.

3 Yao AC, Moinian M, Lind J. Distribution of blood between infant and placenta after birth. Lancet. 1969 Oct;2(7626):871-3.
4 Andersson O, Hellström-Westas L, Andersson D, Domellöf M. Effect of delayed versus early umbilical cord clamping on neonatal outcomes and iron status at 4 months: a randomised controlled trial. BMJ. 2011 Nov;343: d7157.

5 McDonald SJ, Middleton P, Dowswell T, Morris PS. Effect of timing of umbilical cord clamping of term infants on maternal and neonatal outcomes. Cochrane Database Syst Rev. 2013 Jul;2013(7):CD004074.
6 Kc A, Rana N, Målqvist M, Jarawka Ranneberg L, Subedi K, Andersson O. Effects of delayed umbilical cord clamping vs. early clamping on anemia in infants at 8 and 12 months: a randomized clinical trial. JAMA Pediatr. 2017 01;171(3):264-70.

7 Andersson O, Lindquist B, Lindgren M, Stjernqvist K, Domellöf M, Hellström-Westas L. Effect of delayed cord clamping on neurodevelopment at 4 years of age: a randomized clinical trial. JAMA Pediatr. 2015 Jul;169(7):631. 
8 Mercer JS, Erickson-Owens DA, Deoni SCL, Dean DC, Collins J, Parker AB, et al. Effects of delayed cord clamping on 4-month ferritin levels, brain myelin content, and neurodevelopment: a randomized controlled trial. J Pediatr. 2018;203:266-e2.

9 Mercer JS, Erickson-Owens DA, Deoni SCL, Dean Iii DC, Tucker R, Parker AB, et al. The effects of delayed cord clamping on 12-month brain myelin content and neurodevelopment: a randomized controlled trial. Am J Perinatol. 2020 Jul.

10 Rana N, Kc A, Målqvist M, Subedi K, Andersson O. Effect of delayed cord clamping of term babies on neurodevelopment at 12 months: a randomized controlled trial. Neonatology. 2019;115(1):36-42.

11 Kc A, Jha AK, Shrestha MP, Zhou H, Gurung A, Thapa J, et al. Trends for neonatal deaths in Nepal (2001-2016) to project progress towards the SDG target in 2030, and risk factor analyses to focus action. Matern Child Health J. 2020 Feb;24(S1):5-14.

12 Central Bureau of Statistics. Nepal multiple indicator cluster survey 2014, final report. Kathmandu, Nepal: Central Bureau of Statistics and UNICEF Nepal; 2015.

13 Steenis LJ, Verhoeven M, Hessen DJ, van Baar AL. Parental and professional assessment of early child development: the ASQ-3 and the Bayley-III-NL. Early Hum Dev. 2015 Mar; 91(3):217-25.

14 Squires J, Bricker D, Potter L. Revision of a parent-completed developmental screening tool: ages and stages questionnaires. J Pediatr Psychol. 1997;22(3):313-28.
15 Noeder MM, Logan BA, Struemph KL, Condon N, Mueller I, Sands B, et al. Developmental screening in children with CHD: ages and stages questionnaires. Cardiol Young. 2017 Oct;27(8):1447-54.

16 Georgieff MK, Brunette KE, Tran PV. Early life nutrition and neural plasticity. Dev Psychopathol. 2015 May;27(2):411-23.

17 Pollitt E, Triana N. Stability, predictive validity, and sensitivity of mental and motor development scales and pre-school cognitive tests among low-income children in developing countries. Food Nutr Bull. 1999 Jan;20(1):4552.

18 Olvera Astivia OL, Forer B, Dueker GL, Cowling $\mathrm{C}$, Guhn $\mathrm{M}$. The ages and stages questionnaire: latent factor structure and growth of latent mean scores over time. Early Hum Dev. 2017;115:99-109.

19 Andersson O, Domellöf M, Andersson D, Hellström-Westas L. Effects of delayed cord clamping on neurodevelopment and infection at four months of age: a randomised trial. Acta Paediatr. 2013 May;102(5):525-31.

20 Andersson O, Domellöf M, Andersson D, Hellström-Westas L. Effect of delayed vs early umbilical cord clamping on iron status and neurodevelopment at age 12 months: a randomized clinical trial. JAMA Pediatr. 2014 Jun;168(6):547-54.

21 Mercer JS, Erickson-Owens DA, Vohr BR, Tucker RJ, Parker AB, Oh W, et al. Effects of placental transfusion on neonatal and 18 month outcomes in preterm infants: a randomized controlled trial. J Pediatr. 2016 Jan; 168:50-e1.
22 Mercer JS, Vohr BR, Erickson-Owens DA, Padbury JF, Oh W. Seven-month developmental outcomes of very low birth weight infants enrolled in a randomized controlled trial of delayed versus immediate cord clamping. J Perinatol. 2010 Jan;30(1):11-6.

23 Isacson $\mathrm{M}$, Gurung R, Basnet $\mathrm{O}$, Andersson $\mathrm{O}, \mathrm{Kc}$ A. Neurodevelopmental outcomes of a randomised trial of intact cord resuscitation. Acta Paediatr. 2021 Feb;110(2):465-72.

24 Rabe H, Sawyer A, Amess P, Ayers S; for the Brighton Perinatal Study Group. Neurodevelopmental outcomes at 2 and 3.5 years for very preterm babies enrolled in a randomized trial of milking the umbilical cord versus delayed cord clamping. Neonatology. 2016;109(2): 113-9.

25 Thorne-Lyman AL, Shrestha M, Fawzi WW, Pasqualino M, Strand TA, Kvestad I, et al. Dietary diversity and child development in the far West of Nepal: a Cohort Study. Nutrients. 2019 Aug;11(8):1799.

26 Boggs D, Milner KM, Chandna J, Black M, Cavallera V, Dua T, et al. Rating early child development outcome measurement tools for routine health programme use. Arch Dis Child. 2019;104(Suppl 1):S22-33.

27 Small JW, Hix-Small H, Vargas-Baron E, Marks KP. Comparative use of the ages and stages questionnaires in low- and middle-income countries. Dev Med Child Neurol. 2019; 61(4):431-43. 